\title{
HARMONIC UNIVALENT FUNCTIONS
}

\author{
J. CLUNIE and T. SHEIL-SMALL
}

\section{Introduction}

1.1. A continuous function $f=u+i v$ defined in a domain $D \subseteq C$ is harmonic in $D$ if $u$ and $v$ are real harmonic in $D$. In any simply connected subdomain of $D$ we can write $f=\bar{g}+h$, where $g, h$ are analytic and $\bar{g}$ denotes the function $z \mapsto \overline{g(z)}$. The Jacobian of $f$ is then given by

$$
J_{f}(z)=\left|h^{\prime}(z)\right|^{2}-\left|g^{\prime}(z)\right|^{2} .
$$

The mapping $z \mapsto f(z)$ is locally $1-1$ if $J_{f}(z) \neq 0$ in $D$. A result of Lewy [6] shows that the converse is true for harmonic mappings, and therefore $z \mapsto f(z)$ is locally 1-1 and sense preserving if, and only if,

$$
\left|g^{\prime}(z)\right|<\left|h^{\prime}(z)\right| \text {. }
$$

We call such mappings locally univalent, and we say $f$ is univalent in $D$ if $z \mapsto f(z)$ is $1-1$ and sense-preserving in $D$. When $f=\bar{g}+h$ as above we call $\bar{g}$ the co-analytic part and $h$ the analytic part of $f$. Notice that, if $f$ is locally univalent, so is its analytic part.

1.2. From (1.1.2) and Liouville's theorem it is easily seen that the univalent harmonic functions from $C$ to $C$ have the form

$$
f(z)=\alpha \bar{z}+\gamma+\beta z
$$

where $|\alpha|<|\beta|$. These affine transformations will play a considerable role in what follows and we observe that they map lines onto lines and ellipses onto ellipses.

The composition $f \circ \varphi$ of a harmonic function $f$ with an analytic function $\varphi$ is harmonic. However, if $\psi$ is analytic, $\psi \circ f$ is not in general harmonic.

1.3. A function $f=\bar{g}+h$ harmonic in $U=\{|z|<1\}$ can be expanded in a series

$$
f\left(r e^{i \theta}\right)=\sum_{-\infty}^{\infty} a_{n} r^{|n|} e^{i n \theta} \quad(0 \leqq r<1),
$$

where

$$
g(z)=\sum_{1}^{\infty} \bar{a}_{-n} z^{n}, \quad h(z)=\sum_{0}^{\infty} a_{n} z^{n} .
$$


We call $a_{n}=a_{n}(f)$ the coefficients of $f$. The class of functions univalent in $U$ and normalised by $a_{0}=0, a_{1}=1$ will be denoted by $S_{H}$. $S_{H}^{0}$ will denote the subclass with $a_{-1}(f)=0$. Note that the familiar analytic class $S$ is contained in $S_{H}^{0}$.

For $f=\bar{g}+h \in S_{H}^{0}$ we have by (1.1.2) and Schwarz's lemma

$$
\left|g^{\prime}(z)\right| \leqq\left|z h^{\prime}(z)\right| \quad(z \in U),
$$

and in particular

$$
\left|a_{-2}\right| \leqq \frac{1}{2}
$$

1.4. If $f \in S_{H}$, then $\left|a_{-1}\right|<1$ and so applying a transformation of the form (1.2.1) we see that

$$
f_{0}=\frac{f-a_{-1} \bar{f}}{1-\left|a_{-1}\right|^{2}}
$$

belongs to $S_{H}^{0}$. We then have

$$
f=f_{0}+a_{-1} \bar{f}_{0} .
$$

This relation will enable us to obtain various properties of $S_{H}$ from $S_{H}^{0}$.

1.5. If $h \in S$ and $|\varepsilon|<1$, then $f=\varepsilon \bar{h}+h \in S_{H}$. We then have $M(r, f)=$ $O\left(1 /(1-r)^{2}\right)(r \rightarrow 1)$. There are functions in $S_{H}$ with larger growth than this including functions which map onto the Koebe cut-plane which have growth that is not $o\left(1 /(1-r)^{3}\right)(r \rightarrow 1)$. This is one of the many differences between $S_{H}$ and $S$. The straightforward boundary correspondence as exhibited for example in Caratheodory's theorem is no longer true in $S_{H}$. Also, it is not true that, if $t \mapsto f\left(e^{\mathrm{it}}\right)$ represents a sense-preserving homeomorphism of the circle onto a Jordan curve $K$, the harmonic extension of $f$ to $U$ is necessarily a univalent mapping onto the domain bounded by $K$. However, a beautiful result of Choquet [1] shows that this is true when $K$ bounds a convex domain. In particular, if $\varphi(t)$ is a continuous increasing function on $[0,2 \pi]$ with $\varphi(2 \pi)=\varphi(0)+2 \pi$, then the mapping

$$
f(z)=\frac{1}{2 \pi} \int_{0}^{2 \pi}\left(\operatorname{Re} \frac{1+z e^{-i t}}{1-z e^{-i t}}\right) e^{i \varphi(t)} d t
$$

is a harmonic automorphism of $U$.

1.6. Choquet's proof rests on two central facts. The first is purely topological, namely that for a continuous extension to $U$ of a circle homeomorphism to be 1-1, it is sufficient to show that it is locally $1-1$. This result depends on a general result of topological degree which is worth stating explicitly. For $f$ continuous on a closed curve $\gamma$, let $d(f, \gamma)$ denote the degree of $f$ on $\gamma$, i.e. the winding number about the origin of the curve $f \circ \gamma$. We then have what amounts to a generalized argument principle: 
(1.6.1) If $f(z)$ is continuous and non-zero in a domain $D$, then for any cycle $\Gamma$ homologous to zero in $D$,

$$
d(f, \Gamma)=\sum_{\gamma \in \Gamma} d(f, \gamma)=0 .
$$

The second observation of Choquet's proof concerns the affine invariance of convex regions which reduces the problem of local univalence to establishing that $a_{1}(f) \neq 0$. This is achieved by neat but simple arguments rather similar to those we use in Section 5, where we will show further that the analytic part of a convex mapping is univalent. We also obtain the sharp coefficient bounds for convex functions in $S_{H}$ and $S_{H}^{0}$.

1.7. In Section 2 we establish the normality of $S_{H}$ making use of quasiconformal methods and we include growth bounds for $M(r, f)$. Section 3 is concerned with convergence properties. We show that the Caratheodory kernel theorem does not extend to $S_{H}^{0}$. We also obtain a theorem on smooth approximation which is applied later to avoid boundary difficulties. In Section 4 we obtain an undoubtedly poor numerical estimate for $A_{2}=\sup \left\{\left|a_{2}(f)\right|: f \in S_{H}\right\}$. We also obtain lower bounds for $|f(z)|$, which yields in particular a $(1 / 16)$-theorem for $S_{H}^{0}$ in analogy to the $(1 / 4)$ theorem for $S$. Section 5 and Section 6 contain specific examples and a number of sharp results on the special classes of convex, close-to-convex and typically real functions. These results suggest the various open questions and conjectures which conclude the paper.

\section{Normality of $S_{H}$}

2.1. Theorem. Let $f \in S_{H}$. There is an absolute constant $\varrho$ satisfying

$$
0<\varrho \leqq \frac{2 \pi \sqrt{6}}{9}<1.72
$$

such that $f(U)$ omits some value on each circle $\{|\omega|=R\}$ with $R \geqq \varrho$. For $f \in S_{H}^{0}$ there is an omitted value $\omega_{0}$ satisfying

$$
\left|\omega_{0}\right| \leqq \varrho_{0} \leqq \frac{2 \pi \sqrt{3}}{9}<1.21
$$

Proof. Suppose that $f \in S_{H}$ attains in $U$ all values $\omega$ satisfying $|\omega| \leqq t$. Denote by $\varphi(\zeta)$ the conformal mapping of $\{|\zeta|<1\}$ onto the simply-connected domain $f^{-1}(\{|\omega|<t\}) \subseteq U$ for which $\varphi(0)=0, \varphi^{\prime}(0)>0$. Then $\varphi$ extends continuously to $\{|\zeta|=1\}$ and the mapping $\zeta \mapsto f(\varphi(\zeta)) / t$ is a sense-preserving homeomorphism of $\{|\zeta| \leqq 1\}$, which is harmonic in $\{|\zeta|<1\}$. Thus

$$
\frac{1}{t} f\left(\varphi\left(e^{i \theta}\right)\right)=e^{i \alpha(\theta)}=\sum_{-\infty}^{\infty} C_{n} e^{i n \theta},
$$

where $\alpha(\theta)$ is a continuous, strictly increasing function with $\alpha(2 \pi)=\alpha(0)+2 \pi$. 
A recent result of Hall [3], which improves an older result of Heinz [4], gives

$$
\frac{1}{2}\left|C_{-1}\right|^{2}+\left|C_{0}\right|^{2}+\frac{1}{2}\left|C_{1}\right|^{2} \geqq \frac{27}{8 \pi^{2}} .
$$

Now for $f$ given by (1.3.1) we have

$$
t C_{-1}=a_{-1} \varphi^{\prime}(0), C_{0}=0, t C_{1}=\varphi^{\prime}(0) .
$$

By Schwarz's lemma $\varphi^{\prime}(0) \leqq 1$, so we deduce that

$$
t \leqq \frac{2 \pi \sqrt{3}}{9}\left(1+\left|a_{-1}\right|^{2}\right)^{1 / 2} .
$$

The results of the theorem follow immediately.

2.2. Definition. For $f \in S_{H}$ we define the conformal associate of $f$ as the conformal mapping $F: U \rightarrow f(U)$ for which $F(0)=0, F^{\prime}(0)>0$.

2.3. Theorem. $S_{H}$ is a normal family and $S_{H}^{0}$ is a compact normal family.

Proof. We obtain an explicit upper bound for $M(r, f)$, where $0 \leqq r<1$ and $f \in S_{H}$. From (1.4.2) we have

$$
M(r, f)<2 M\left(r, f_{0}\right),
$$

where $f_{0} \in S_{H}^{0}$. We suppose now that $f=\bar{g}+h \in S_{H}^{0}$. Then for $|z| \leqq R<1$ we have $\left|g^{\prime}(z)\right| \leqq R\left|h^{\prime}(z)\right|$ and so the mapping $z \rightarrow f(R z)(z \in U)$ is $K_{R}$-quasiconformal, where $K_{R}=(1+R) /(1-R)$.

Put $\omega(z)=F^{-1}(f(z))(z \in U)$, where $F$ is defined in (2.2). Then the mapping $z \mapsto \omega(R z) \quad(z \in U)$ is $K_{R}$-quasiconformal in $U$ and satisfies $|\omega(R z)|<1, \omega(0)=0$. By a well-known distortion theorem [5, p. 64]

$$
|\omega(R z)| \leqq \varphi_{K_{R}}(|z|) \quad(z \in U) .
$$

Here

$$
\varphi_{K}(r)=\mu^{-1}\left(\frac{\mu(r)}{K}\right) \quad(0<r<1),
$$

where $\mu(r)$ denotes the module of the domain consisting of the unit disc cut from 0 to $r$ along the positive real axis. We refer to [5], Chapter II for the properties of $\mu(r)$ and $\varphi_{K}(r)$. For the moment it is sufficient to note that $\varphi_{K}(r)$ is a strictly increasing mapping of $[0,1]$ into itself for each fixed $K>0$. From (2.3.2) we see that

$$
\begin{aligned}
\left|\omega\left(r e^{i \theta}\right)\right| \leqq \sigma(r) & =\inf \left\{\varphi_{K_{R}}\left(\frac{r}{R}\right): r<R<1\right\} \\
& =\inf \left\{\varphi_{K}\left(\frac{K+1}{K-1} r\right): K>\frac{1+r}{1-r}\right\} .
\end{aligned}
$$


Clearly $\sigma(r)$ is a strictly increasing mapping of $[0,1]$ into itself. Now $f(z)=F(\omega(z))$ and hence from the Koebe distortion theorem

$$
M(r, f) \leqq \frac{F^{\prime}(0) \sigma(r)}{(1-\sigma(r))^{2}} .
$$

Furthermore $f(U)=F(U)$ and so by Theorem 2.1 and the (1/4)-theorem

$$
F^{\prime}(0) \leqq 4 \varrho_{0} \leqq \frac{8 \pi \sqrt{3}}{9} \text {. }
$$

This establishes normality, since a family of harmonic functions is normal if it is locally bounded.

2.4. To prove compactness of $S_{H}^{0}$ let $\left(f_{n}\right)=\left(\bar{g}_{n}+h_{n}\right)$ be a sequence in $S_{H}^{0}$ converging locally uniformly to $f=\bar{g}+h$. By Hurwitz's theorem $h^{\prime}(z)=\lim _{n} h_{n}^{\prime}(z) \neq 0$ in $U$. Also $\left|g^{\prime}(z) / h^{\prime}(z)\right| \leqq|z|$, so $f$ is locally univalent.

Therefore, if $z_{1}$ and $z_{2} \in U, z_{1} \neq z_{2}$, we have $d\left(f-f\left(z_{1}\right), C_{1}\right)>0, d\left(f-f\left(z_{2}\right), C_{2}\right)>0$ for sufficiently small circles $C_{1}, C_{2}$ about $z_{1}, z_{2}$ respectively. Since

$$
\frac{f_{n}(z)-f\left(z_{1}\right)}{f(z)-f\left(z_{1}\right)} \rightarrow 1 \text { uniformly on } C_{1},
$$

$d\left(f_{n}-f\left(z_{1}\right), C_{1}\right)=d\left(f-f\left(z_{1}\right), C_{1}\right)$ for large $n$. By the degree principle (1.6.1), $f_{n}$ attains the value $f\left(z_{1}\right)$ in the disc bounded by $C_{1}$. Similarly $f_{n}$ attains the value $f\left(z_{2}\right)$ near $z_{2}$ and therefore the univalence of $f_{n}$ implies $f\left(z_{1}\right) \neq f\left(z_{2}\right)$. Thus $f$ is univalent in $U$.

2.5. Theorem. The closure $\bar{S}_{H}$ of $S_{H}$ consists of all functions of the form

$$
f=f_{0}+\varepsilon \bar{f}_{0}
$$

where $|\varepsilon| \leqq 1$ and $f_{0} \in S_{H}^{0}$. Also $f \in \partial S_{H}=\bar{S}_{H} \backslash S_{H}$ if, and only if, $|\varepsilon|=1$. Every extreme point of the closed convex hull of $\bar{S}_{H}$ lies on $\partial S_{H}$ and has the form

$$
f=f_{0}+e^{i \alpha} \bar{f}_{0},
$$

where $f_{0}$ is an extreme point of $S_{H}^{0}$.

Proof. The first two statements follow from (1.4.2) and the compactness of $S_{H}^{0}$. Let $f$ be an extreme point of the hull of $\bar{S}_{H}$, so $f \in \bar{S}_{H}$ by the Krein-Milman theorem. Hence $f=f_{0}+r e^{i \alpha} \bar{f}_{0}$, where $0 \leqq r \leqq 1$ and $f_{0} \in S_{H}^{0}$. Then

$$
f=\frac{1}{2}(1+r)\left(f_{0}+e^{i \alpha} \bar{f}_{0}\right)+\frac{1}{2}(1-r)\left(f_{0}-e^{i \alpha} \bar{f}_{0}\right)
$$

is a convex combination of elements in $\bar{S}_{H}$ and so we must have $r=1$. Finally, if $f_{0}$ were not an extreme point of $S_{H}^{0}$, then we could write

$$
f_{0}=(1-t) p_{0}+t q_{0}
$$


where $0<t<1$ and $p_{0}, q_{0} \in S_{H}^{0}$. But then

$$
f=(1-t)\left(p_{0}+e^{i \alpha} \bar{p}_{0}\right)+t\left(q_{0}+e^{i \alpha} \bar{q}_{0}\right)
$$

would not be an extreme point.

2.6. Remark. Theorem 2.5 will clearly hold for any subclass of $S_{H}$ which is invariant under the transformations (1.2.1) suitably normalized, provided that the corresponding subclass of $S_{H}^{0}$ is compact. The relationship between compact classes in $S$ and $S_{H}^{0}$ is taken up in the next section.

\section{Convergence properties}

3.1. In establishing the compactness of various subclasses of $S_{H}^{0}$ proofs are complicated by the fact that the Caratheodory kernel theorem is not valid in $S_{H}^{0}$. The difficulty arises essentially out of the possibility of discontinuities in the boundary function. It will be recalled that a sequence $\left(F_{n}\right)$ of functions in $S$ converges to $F \in S$ (i.e. locally uniformly) if, and only if, the sequence of domains $\left(F_{n}(U)\right)$ converges in the sense of kernel convergence to $F(U)$. This result fails in $S_{H}^{0}$ both for the necessity and the sufficiency.

3.2. Example. Define

$$
f(z)=\frac{1}{2 \pi} \int_{0}^{2 \pi}\left(\operatorname{Re} \frac{1+z e^{-i t}}{1-z e^{-i t}}\right) e^{i \varphi(t)} d t \quad(z \in U),
$$

where

$$
\varphi(t)=\frac{2 \pi k}{3} \quad\left(\frac{2 \pi k}{3} \leqq t<\frac{2 \pi(k+1)}{3}, \quad k=0,1,2\right) .
$$

For this function we have

$$
a_{0}(f)=a_{-1}(f)=0, a_{1}(f)=\frac{3}{2 \pi i}\left(1-e^{-2 \pi i / 3}\right) .
$$

Clearly $f(z)$ for $z \in U$ takes no values outside the triangle whose vertices are the cube roots of unity. Furthermore, by the following reasoning $f$ is univalent in $U$.

Since $\varphi(t)$ is a non-decreasing step function in $[0,2 \pi]$ it is almost everywhere the pointwise limit of a sequence $\left(\varphi_{n}(t)\right)$ of strictly increasing, continuous functions on $[0,2 \pi]$ satisfying $\varphi_{n}(2 \pi)=\varphi_{n}(0)+2 \pi$. The functions

$$
f_{n}(z)=\frac{1}{2 \pi} \int_{0}^{2 \pi}\left(\operatorname{Re} \frac{1+z e^{-i t}}{1-z e^{-i t}}\right) e^{i \varphi_{n}(t)} d t \quad(z \in U)
$$

then form a sequence which converges locally uniformly in $U$ to $f(z)$. By Choquet's theorem each $f_{n}(z)$ is an automorphism of $U$. Thus $f$ is univalent in $U$ and one can check that $f(U)$ is the inside of the above triangle. Since $f_{n}(U)=U(n \geqq 1)$ one sees, 
after suitable normalizations, that one can have local uniform convergence without having kernel convergence.

3.3. It is also not the case that for a sequence $\left(f_{n}\right)$ in $S_{H}^{0}, f_{n}(U) \rightarrow f(U)$ implies $f_{n} \rightarrow f$. This follows immediately from the fact that there exist $f_{1}, f_{2} \in S_{H}^{0}$ with $f_{1}(U)=$ $f_{2}(U)$, but $f_{1} \neq f_{2}$. For example, we can take

$$
f_{1}(z)=\frac{z}{1-z}, \quad f_{2}(z)=l_{0}(z),
$$

where $l_{0}(z)$ is defined in (5.5). Both functions are in $S_{H}^{0}$ and each maps $U$ onto $\{\operatorname{Re} w>-1 / 2\}$.

3.4. In Example 3.2 we note the following: each $f_{n}(U)$ is convex, $f(U)$ is convex and $f(U)$ is obtained from the kernel of $\left(f_{n}(U)\right)$ by making certain cross-cuts. Such properties can be associated with convergence in a more general setting. This will be discussed in detail in a forthcoming paper, where it will be shown that certain subclasses of $S_{H}^{0}$, corresponding to classical geometrically defined subclasses of $S$, are compact. The convex case will be considered in Section 5 by a direct argument. For now we prove only the following weaker result.

3.5. Theorem. Let $\left(f_{n}\right)$ be a sequence of functions in $S_{H}^{0}$ converging locally uniformly in $U$ to $f$ and let $\Omega$ be the kernel of $\left(f_{n}(U)\right)$ with respect to 0 . Then

$$
f(U) \subseteq \Omega \text {. }
$$

Proof. If $0<r<\varrho<1$, then, because $f$ is univalent,

$$
\frac{f_{n}(z)-f\left(z_{0}\right)}{f(z)-f\left(z_{0}\right)} \rightarrow 1 \text { as } n \rightarrow \infty
$$

uniformly for $|z|=\varrho,\left|z_{0}\right| \leqq r$. Therefore for $n>m$ say, the expression (3.5.2) has positive real part when $|z|=\varrho,\left|z_{0}\right| \leqq r$. It follows from the degree principle that, if $n>m, f_{n}(z)$ attains each value $f\left(z_{0}\right)\left(\left|z_{0}\right| \leqq r\right)$ for some $z$ in $|z|<\varrho$ and so

$$
f\left(\left|z_{0}\right| \leqq r\right) \subseteq \bigcap_{n=m}^{\infty} f_{n}(U) .
$$

The result follows immediately.

3.6. If $f$ is harmonic univalent in $U$ we say that $f_{0}$ is subordinate to $f$ if we can write

$$
f_{0}(z)=f(\omega(z))
$$

where $\omega(z)$ is analytic univalent in $U$ with $|\omega(z)|<1$ in $U$ and

$$
\omega(0)=0, \quad \omega^{\prime}(0)>0 .
$$

It is clear that, if $D_{0}$ is a simply-connected domain with $f(0) \in D_{0} \subseteq f(U)$, then there is a unique function $f_{0}(z)$ subordinate to $f(z)$ with $f_{0}(U)=D_{0}$. Indeed we define $\omega(z)$ as the conformal mapping of $U$ onto $f^{-1}\left(D_{0}\right)$ satisfying (3.6.2). 
3.7. Approximation theorem. Let $f$ be harmonic univalent in $U$ and let $\left(D_{k}\right)$ be a sequence of simply-connected domains such that $f(0) \in D_{k} \subseteq f(U)(k=1,2, \ldots)$ and such that

$$
D_{k} \rightarrow f(U) \quad(k \rightarrow \infty)
$$

in the sense of kernel convergence. Then the sequence of subordinate functions $\left(f_{k}\right)$ converges locally uniformly to $f$ in $U$. Furthermore, if each domain $D_{k}$ is bounded by a Jordan curve $J_{k} \subseteq D$, then $f_{k}$ extends continuously to give a homeomorphism of $\bar{U}$ onto $\bar{D}_{k}$. If each $\bar{J}_{k}$ is smooth then $f_{k}$ extends smoothly to $\bar{U}$.

Proof. Let $f_{k}=f \circ \omega_{k}$. If $0<r<1, f(\{|z| \leqq r\})$ is a compact subset of $f(U)$ and so there is a $K$ such that

$$
f(\{|z| \leqq r\}) \subseteq D_{k} \quad(k>K) .
$$

Hence $\{|z| \leqq r\} \subseteq f^{-1}\left(D_{k}\right)=\omega_{k}(U)$ and so $\left(\omega_{k}(U)\right)$ converges to its kernel $U$. By Caratheodory's kernel theorem, $\omega_{k}(z) \rightarrow z$ locally uniformly in $U$, and hence the same is true for $f_{k} \rightarrow f$. If $D_{k}$ is bounded by a Jordan curve $J_{k} \subseteq f(U)$, then $\omega_{k}(U)$ is bounded by a Jordan curve $f^{-1}\left(J_{k}\right) \subseteq U$, so $\omega_{k}$ extends continuously to a homeomorphism of $\bar{U}$ onto $\overline{\omega_{k}(U)}$; and similarly in the case of smoothness.

\section{Numerical estimates and a distortion theorem}

4.1. Theorem. Let $f \in S_{H}$, Then

$$
\left|a_{2}(f)\right|<12,173 \text {. }
$$

Proof. Suppose at first that $f \in S_{H}^{0}$. Then from (2.3.5) and (2.3.6) we have

$$
M(r, f) \leqq \frac{8 \pi \sqrt{3}}{9} \frac{\sigma(r)}{(1-\sigma(r))^{2}} \quad(0 \leqq r<1),
$$

where $\sigma(r)$ is given by (2.3.4). We choose $K=2$, when

$$
\varphi_{2}(r)=\frac{2 \sqrt{r}}{1+r} \quad(\text { see }[5], \text { p. 64) }
$$

We then have

$$
\begin{aligned}
\left|a_{2}(f)\right| & \leqq \frac{M(r, f)}{r^{2}} \leqq \frac{8 \pi \sqrt{3}}{9 r^{2}} \frac{\varphi_{2}(3 r)}{\left(1-\varphi_{2}(3 r)\right)^{2}} \quad(0<r<1 / 3) \\
& =8 \pi \sqrt{3} \frac{\varphi_{2}(t)}{t^{2}\left(1-\varphi_{2}(t)\right)^{2}} \quad(0<t<1) \\
& =16 \pi \sqrt{3} \frac{1+t}{t^{3 / 2}\left(1-t^{1 / 2}\right)^{4}}=16 \pi \sqrt{3} \frac{1+S^{2}}{S^{3}(1-S)^{4}} \quad(0<S<1) .
\end{aligned}
$$


Choosing $S=.4046385951$ we obtain

$$
\left|a_{2}(f)\right|<12,172 .
$$

For general $f \in S_{H}$ we need to add at most $1 / 2$ to this estimate from (1.4.2) and (1.3.4).

4.2. Bounds for $\left|a_{2}(f)\right|$ lead to distortion results for $f \in S_{H}$ just as in the analytic case. Writing

$$
A_{2}=\sup \left\{\left|a_{2}(f)\right|: f \in S_{H}\right\}
$$

we note that if $f=\bar{g}+h \in S_{H}$, then the function

$$
\varphi(z)=\frac{f\left(\left(z+z_{0}\right) /\left(1+\bar{z}_{0} z\right)\right)-f\left(z_{0}\right)}{\left(1-\left|z_{0}\right|^{2}\right) h^{\prime}\left(z_{0}\right)}
$$

is also in $S_{H}$, if $\left|z_{0}\right|<1$. We deduce that

$$
\left|\frac{1}{2}\left(1-\left|z_{0}\right|^{2} h^{\prime \prime}\left(z_{0}\right)-\bar{z}_{0}\left(1-\left|z_{0}\right|^{2}\right) h^{\prime}\left(z_{0}\right)\right)\right| \leqq A_{2}\left(1-\left|z_{0}\right|^{2}\right)\left|h^{\prime}\left(z_{0}\right)\right|
$$

from which it follows that

$$
\frac{(1-|z|)^{A_{2}-1}}{(1+|z|)^{A_{2}+1}} \leqq\left|h^{\prime}(z)\right| \leqq \frac{(1+|z|)^{A_{2}-1}}{(1-|z|)^{A_{2}+1}} \quad(|z|<1) .
$$

From $\left|g^{\prime}(z)\right|<\left|h^{\prime}(z)\right|$ it follows that

$$
|f(z)| \leqq 2 \int_{0}^{r} \frac{(1+t)^{A_{2}-1}}{(1-t)^{A_{2}+1}} d t \quad(|z|=r<1) .
$$

In particular we see that

$$
|f(z)|=0\left(\frac{1}{(1-|z|)^{A_{2}}}\right) \quad(|z| \rightarrow 1) .
$$

4.3. There can be no overall positive lower bound for $|f(z)|$ depending on $|z|$, when it is known only that $f \in S_{H}$ since, for example $z+\varepsilon \bar{z} \in S_{H}$ for all $\varepsilon$ with $|\varepsilon|<1$. However, a lower bound can be obtained depending on $\left|a_{-1}(f)\right|$.

4.4. Theorem. For $f \in S_{H}^{0}$ we have

$$
|f(z)| \geqq \frac{1}{4} \frac{|z|}{(1+|z|)^{2}} \quad(|z|<1) .
$$

\section{In particular}

$$
d=\sup \left\{\varrho:\{|w|<\varrho\} \subseteq f(U), \quad f \in S_{H}^{0}\right\} \geqq \frac{1}{16} .
$$

Proof. For $0<\sigma<1$ let $S_{H}^{0}(\sigma)$ denote the class of functions $f$ of the form

$$
f(z)=\frac{F(\sigma z)}{\sigma} \quad\left(F \in S_{H}^{0}\right) .
$$


Suppose that $f \in S_{H}^{0}(\sigma)$ and let $D=f(U), D_{\varepsilon}=D \backslash\{|w| \leqq \varepsilon\}$ for small $\varepsilon>0$ such that $\{|w| \leqq \varepsilon\} \subseteq D$. Let $\delta$ be the distance from 0 to $\partial f(U)$ and assume without loss of generality that $-\delta \in \partial f(U)$. Let $\Delta$ be the plane cut along the negative real axis from $-\delta$ to $-\infty$ and let $\Delta_{\varepsilon}=\Delta \backslash\{|w| \leqq \varepsilon\} . D_{\varepsilon}$ and $\Delta_{\varepsilon}$ are ring domains and

$$
M\left(D_{\varepsilon}\right) \leqq M\left(\Delta_{\varepsilon}\right),
$$

where $M$ denotes module.

From the normalisation $D_{\varepsilon}$ is approximately the image of the ring $R_{\varepsilon}=$ $\{\varepsilon<|z|<1\}$ by $f$ for small $\varepsilon>0$ and it is easily checked that the approximation is sufficient to justify the following arguments as $\varepsilon \rightarrow 0$.

If $\varrho$ is an admissible metric for $R_{\varepsilon}$, let $\varrho$ be a metric for $D_{\varepsilon}$ defined by

$$
\tilde{\varrho}(w)=\frac{\varrho\left(f^{-1}(w)\right)}{\left|h^{\prime}(z)\right|-\left|g^{\prime}(z)\right|},
$$

where $f=\bar{g}+h, w=f(z)$. Let $\gamma$ be a path joining $\{|z|=\varepsilon\}$ to $\{|z|=1\}$ in $R_{\varepsilon}$ and let $\tilde{\gamma}$ be its image under $f$ in $D_{\varepsilon}$. Then

$$
\int_{\tilde{\gamma}} \tilde{\varrho}(w)|d w|=\int_{\gamma} \frac{\varrho(z)\left|\partial_{\alpha} f(z)\right|}{\left|h^{\prime}(z)\right|-\left|g^{\prime}(z)\right|}|d z|,
$$

where $\partial_{\alpha} f(z)$ is the appropriate directional derivative. Now $\left|\partial_{\alpha} f(z)\right| \geqq\left|h^{\prime}(z)\right|-\left|g^{\prime}(z)\right|$ and so

$$
\int_{\tilde{\gamma}} \tilde{\varrho}(w)|d w| \geqq \int_{\gamma} \varrho(z)|d z| \geqq 1,
$$

and therefore $\tilde{\varrho}(w)$ is admissible for $D_{\varepsilon}$. Hence

$$
\begin{aligned}
& \frac{1}{M\left(D_{\varepsilon}\right)} \leqq \inf _{\tilde{\varrho}} \iint_{D_{\varepsilon}} \tilde{\varrho}(w)^{2} d u d v \quad(w=u+i v) \\
= & \inf _{\varrho} \iint_{R_{\varepsilon}} \varrho^{2}(z) \frac{J_{f}(z)}{\left(\left|h^{\prime}(z)\right|-\left|g^{\prime}(z)\right|\right)^{2}} d x d y \quad(z=x+i y) \\
\leqq & \inf _{\varrho} \iint_{R_{\varepsilon}} \frac{1+\sigma|z|}{1-\sigma|z|} \varrho^{2}(z) d x d y,
\end{aligned}
$$

the last inequality following from $\left|g^{\prime}(z)\right| \leqq \sigma|z| \cdot\left|h^{\prime}(z)\right|$ for $f \in S_{H}^{0}(\sigma)$. We choose

$$
\varrho(z)=\frac{(1-\sigma r) /((1+\sigma r) r)}{\int_{\varepsilon}^{1}((1-\sigma t) /(1+\sigma t) t) d t} \quad\left(z=r e^{i \theta}\right)
$$

and perform the integration in (4.4.6) to obtain

and hence

$$
\frac{1}{M\left(D_{\varepsilon}\right)} \leqq 2 \pi\left(\log \frac{1}{\varepsilon}-2 \log (1+\sigma)+2 \log (1+\sigma \varepsilon)\right)^{-1}
$$

$$
M\left(D_{\varepsilon}\right) \geqq \frac{1}{2 \pi}\left(\log \frac{1}{\varepsilon}-2 \log (1+\sigma)\right) .
$$


On the other hand $4 \delta z(1-z)^{-2}$ maps $U$ onto $\Delta$ and approximately maps $R_{\varepsilon / 4 \delta}$ onto $\Delta_{\varepsilon}$. Since the module is a conformal invariant, we see that

$$
M\left(\Delta_{\varepsilon}\right) \simeq M\left(R_{\varepsilon / 4 \delta}\right)=\frac{1}{2 \pi} \log \left(\frac{4 \delta}{\varepsilon}\right) .
$$

From (4.4.4), (4.4.9) and (4.4.10) we see by letting $\varepsilon \rightarrow 0$ that

$$
-2 \log (1+\sigma) \leqq \log 4 \delta \text {. }
$$

We deduce that for $f \in S_{H}^{0}(\sigma)$,

$$
|f(z)| \geqq \delta \geqq \frac{1}{4} \frac{1}{(1+\sigma)^{2}} \quad(|z|=1) .
$$

We immediately obtain (4.4.1) for $f \in S_{H}^{0}$.

4.5. Corollary. Let $f \in S_{H}$. Then

$$
|f(z)| \geqq \frac{1}{4}\left(1-\left|a_{-1}(f)\right|\right) \frac{|z|}{(1+|z|)^{2}} \quad(|z|<1) .
$$

Also

$$
\left(|w|<\frac{1}{16}\left(1-\left|a_{-1}(f)\right|\right)\right) \leqq f(U) .
$$

\section{Convex and close-to-convex mappings}

5.1. Let $K, K_{H}$ and $K_{H}^{0}$ denote the respective subclasses of $S, S_{H}$ and $S_{H}^{0}$ where the images $f(U)$ are convex. Also let $C, C_{H}$ and $C_{H}^{0}$ be the subclasses with close-to-convex images. We recall that a domain $D$ is close-to-convex if the complement of $D$ can be written as a union of non-crossing half-lines. For other equivalent criteria see [9]. Every star-shaped domain is close-to-convex. A domain $D$ is called convex in the direction $\varphi(0 \leqq \varphi<\pi)$ if every line parallel to the line through 0 and $e^{i \varphi}$ has a connected intersection with $D$. Such a domain is close-to-convex. The convex domains are those convex in every direction. The following lemma will enable us to construct examples of harmonic convex and close-to-convex mappings.

5.2. Lemma. Let $D$ be a domain convex in the direction of the real axis and let $p(w)$ be a continuous real-valued function in $D$. Then the mapping

$$
w \rightarrow w+p(w)
$$

is 1-1 in D if, and only if, the mapping is locally 1-1. In this case the image of D is convex in the direction of the real axis.

Proof. If $w_{1}+p\left(w_{1}\right)=w_{2}+p\left(w_{2}\right) \quad\left(w_{1} \neq w_{2}\right)$, then writing $w_{1}=u_{1}+i v_{1}, w_{2}=$ $u_{2}+i v_{2}$ we have $v_{1}=v_{2}=c$, say, and $u_{1}+p\left(u_{1}+i c\right)=u_{2}+p\left(u_{2}+i c\right)$. Therefore the real function $u \rightarrow u+p(u+i c)$, which is defined on some interval $\left(\alpha_{c}, \beta_{c}\right)$, is not strictly monotonic and therefore not locally $1-1$. 
The image of $D$ is convex in the direction of the real axis, since each line segment $w=u+i c\left(\alpha_{c}<u<\beta_{c}\right)$ is mapped onto an interval.

5.3. Theorem. A harmonic $f=\bar{g}+h$ locally univalent in $U$ is a univalent mapping of $U$ onto a domain convex in the direction of the real axis if, and only if, $h-g$ is a conformal univalent mapping of $U$ onto a domain convex in the direction of the real axis. In particular the subclass of $S_{H}^{0}$ consisting of functions $f$ such that $f(U)$ is convex in the direction of the real axis is compact.

Proof. If $f$ is univalent and $f(U)$ is convex in the direction of the real axis, then writing $w=f(z), z=z(w)$, we have

$$
h(z(w))-g(z(w))=w-2 \operatorname{Re} g(z(w))=w+p(w) .
$$

Since $h^{\prime}(z)-g^{\prime}(z) \neq 0$ in $U$ and $z(w)$ is $1-1$, the mapping $w \rightarrow w+p(w)$ is locally $1-1$ and so univalent by Lemma 5.2. Hence $z \rightarrow h(z)-g(z)$ is univalent. The compactness result follows easily from the compactness of the corresponding subclass in $S$.

Conversely, if $h-g$ is convex in the direction of the real axis, then writing $W=h(z)-g(z), z=z(W)$, we have

$$
f(z(w))=W+2 \operatorname{Re} g(z(W))=W+q(W)
$$

is locally 1-1 and so univalent by Lemma 5.2 .

5.4. Example. Since $z /(1-z)^{2}$ is convex in the direction of the real axis, so is the harmonic function $k_{0}=\bar{g}+h$, where

$$
h(z)-g(z)=\frac{z}{(1-z)^{2}}, \quad g^{\prime}(z)=z h^{\prime}(z)
$$

We obtain

$$
\begin{gathered}
h^{\prime}(z)=\frac{1+z}{(1-z)^{4}}, \quad g^{\prime}(z)=\frac{z(1+z)}{(1-z)^{4}} \\
h(z)=\frac{z-(1 / 2) z^{2}+(1 / 6) z^{3}}{(1-z)^{3}}, \quad g(z)=\frac{(1 / 2) z^{2}+(1 / 6) z^{3}}{(1-z)^{3}} \\
k_{0}(z)=\operatorname{Re}\left(\frac{z+(1 / 3) z^{3}}{(1-z)^{3}}\right)+i \operatorname{Im}\left(\frac{z}{(1-z)^{2}}\right) .
\end{gathered}
$$

Hence $k_{0} \in C_{H}^{0}$ and we now show that $k_{0}(U)$ is $\mathbf{C}$ cut along the negative real axis from $-1 / 6$ to $\infty$. We write $(1+z) /(1-z)=w=u+i v$, when (5.4.4) becomes

$$
\frac{1}{6} \operatorname{Re}\left(w^{3}-1\right)+\frac{1}{4} i \operatorname{Im}\left(w^{2}\right)=\frac{1}{6}\left(u^{3}-3 u v^{2}-1\right)+\frac{1}{2} i u v,
$$

where $u>0$. If $u v=0$, the right hand side of (5.4.5) varies between $-1 / 6$ and $+\infty$. On the level curve $u v=c \neq 0$, the real part is $u^{3} / 6-c^{2} /(2 u)$, which varies between $-\infty$ and $+\infty \quad(0<u<+\infty)$. 
Note that the function

$$
k(z)=2 \operatorname{Re}(g(z)+h(z))=2 \operatorname{Re}\left(\frac{z+(1 / 3) z^{3}}{(1-z)^{3}}\right)
$$

is in $\partial C_{H} \subseteq \partial S_{H}$.

5.5. Example. Since $z /(1-z)$ is convex, the function $l_{0}=\bar{g}+h$ defined by

$$
h(z)+g(z)=\frac{z}{1-z}, \quad g^{\prime}(z)=-z h^{\prime}(z)
$$

is convex in the direction of the imaginary axis. We obtain

$$
\begin{gathered}
h^{\prime}(z)=\frac{1}{(1-z)^{3}}, \quad g^{\prime}(z)=-\frac{z}{(1-z)^{3}} \\
h(z)=\frac{z-(1 / 2) z^{2}}{(1-z)^{2}}, \quad g(z)=-\frac{(1 / 2) z^{2}}{(1-z)^{2}} \\
l_{0}(z)=\operatorname{Re}\left(\frac{z}{1-z}\right)+i \operatorname{Im}\left(\frac{z}{(1-z)^{2}}\right)
\end{gathered}
$$

It is easily seen that $l_{0}(U)=\{\operatorname{Re} w>-1 / 2\}$, so $l_{0} \in K_{H}^{0}$.

The function

$$
l(z)=2 i \operatorname{Im}\left(\frac{z}{(1-z)^{2}}\right)
$$

is in $\partial K_{H}$.

5.6. Remark. Note that

$$
l_{0}\left(e^{i \theta}\right)=\lim _{r \rightarrow 1} l_{0}\left(r e^{i \theta}\right)=-\frac{1}{2} \quad(0<\theta<2 \pi)
$$

so even with such a smooth boundary as $\{\operatorname{Re} w=-1 / 2\}$ the boundary correspondence which holds in the conformal case fails in the harmonic case. Furthermore it can be shown that $l_{0}(z)$ does not map the circle $\{|z|=r\}$ onto a convex curve if $\sqrt{2}-1<r<1$. Thus the hereditary property for convex conformal maps fails in the harmonic case. This corresponds to the failure of the hereditary property for analytic functions convex in one direction [2].

5.7. Theorem. A function $f=\bar{g}+h \in K_{H}$ if, and only if, the analytic functions

$$
h(z)-e^{i \varphi} g(z) \quad(0 \leqq \varphi<2 \pi)
$$

are convex in the direction $\varphi / 2$ and $f$ is suitably normalized. In this case the functions

$$
h(z)+\varepsilon g(z) \quad(|\varepsilon| \leqq 1)
$$


are close-to-convex in $U$. In particular $h$ is close-to-convex. Finally $K_{H}^{0}$ is a compact class.

Proof. If $f$ is convex, then $e^{-i \varphi / 2} f=e^{-i \varphi / 2} h+\overline{e^{i \varphi / 2} g}$ is convex, so $e^{-i \varphi / 2} h-e^{i \varphi / 2} g$ is convex in the direction of the real axis by Theorem 5.3. Hence $h-e^{i \varphi} g$ is convex in the direction $\varphi / 2$. Conversely, the latter implies that $f$ is convex in each direction $\varphi / 2$, so $f$ is convex. In particular $h+\varepsilon g$ is close-to-convex when $|\varepsilon|=1$ and hence for $0<r<1$ and $\theta_{1}<\theta_{2}<\theta_{1}+2 \pi$,

$$
\int_{\theta_{1}}^{\theta_{2}} \operatorname{Re}\left(1+\frac{r e^{i \theta} h^{\prime \prime}\left(r e^{i \theta}\right)+\varepsilon r e^{i \theta} g^{\prime \prime}\left(r e^{i \theta}\right)}{h^{\prime}\left(r e^{i \theta}\right)+\varepsilon g^{\prime}\left(r e^{i \theta}\right)}\right) d \theta>-\pi .
$$

As the expression on the left is harmonic in $\varepsilon$ for $|\varepsilon| \leqq 1$, this inequality continues to hold when $|\varepsilon| \leqq 1$. This proves (5.7.2). The compactness of $K_{H}^{0}$ follows from Theorem 5.3.

5.8. Corollary. If $f=\bar{g}+h \in K_{H}$, then

$$
\left|\frac{g\left(z_{1}\right)-g\left(z_{2}\right)}{h\left(z_{1}\right)-h\left(z_{2}\right)}\right|<1 \quad\left(\left|z_{1}\right|,\left|z_{2}\right|<1\right) .
$$

In particular, if $f \in K_{H}$,

$$
|g(z)|<|h(z)| \quad(0<|z|<1) .
$$

Proof. From (5.7.2) we deduce that

$$
\frac{h\left(z_{1}\right)-h\left(z_{2}\right)}{z_{1}-z_{2}}+\varepsilon \frac{\left(g\left(z_{1}\right)-g\left(z_{2}\right)\right)}{z_{1}-z_{2}} \neq 0
$$

for $|\varepsilon| \leqq 1,\left|z_{1}\right|,\left|z_{2}\right|<1$ and (5.8.1) follows immediately.

5.9. Theorem. If $f \in K_{H}^{0}$, then

$$
\left(|w|<\frac{1}{2}\right) \subseteq f(U) .
$$

Proof. If $w \notin f(U)$, then for suitable real $\mu$,

$$
\left.\operatorname{Re}\left(e^{i \mu}(f(z)-w)\right)\right)>0 \quad(|z|<1) .
$$

Hence if $f=\bar{g}+h$,

$$
\operatorname{Re}\left(e^{i \mu}(h(z)-w)+e^{-i \mu} g(z)\right)>0 \quad(|z|<1) .
$$

Thus applying a standard inequality for the coefficient of $z$ we obtain $\left|e^{-i \mu}\right| \leqq 2\left|w e^{i \mu}\right|$, i.e. $|w| \geqq 1 / 2$. 
5.10. Theorem. If $f \in K_{H}^{0}$, then for $n=1,2, \ldots$ we have

$$
|| a_{n}(f)|-| a_{-n}(f)|| \leqq 1 ;
$$

$$
\left|a_{-n}(f)\right| \leqq \frac{n-1}{2} ;
$$

$$
\left|a_{n}(f)\right| \leqq \frac{n+1}{2} .
$$

Equality occurs for $f=l_{0}$ of Example 5.5.

5.11. Lemma. If $f=\bar{g}+h \in K_{H}$, then there exist real $\lambda$, $\mu$ such that

$$
\operatorname{Re}\left(\left(e^{-i \mu} h^{\prime}(z)+e^{i \mu} g^{\prime}(z)\right)\left(e^{i \lambda}-e^{-i \lambda} z^{2}\right)\right) \geqq 0 \quad(|z|<1) .
$$

Proof. Applying Approximation theorem 3.7 we may assume that $f$ extends smoothly to $\{|z|=1\}$ with $f\left(e^{i \theta}\right)$ describing a smooth convex curve. Then

$$
l(\theta)=\frac{\partial}{\partial \theta} f\left(e^{i \theta}\right)
$$

is continuous, periodic with period $2 \pi$ and $\neq 0$. Furthermore $\arg l(\theta)$ exists continuously and is increasing, so that $l(\theta)(0 \leqq \theta \leqq 2 \pi)$ describes a continuous curve starlike with respect to 0 and rotating once about 0 in the positive direction. It follows that for each real $t$ we can find $t^{*}\left(t<t^{*}<t+2 \pi\right)$ such that

$$
\frac{l\left(t^{*}\right)}{\left|l\left(t^{*}\right)\right|}=-\frac{l(t)}{|l(t)|} \text {. }
$$

Furthermore $t^{*}$ varies continuously with $t$ and $t^{* *}=t+2 \pi$. Therefore there is a $t_{0}$ with $t_{0}^{*}=t_{0}+\pi$. We thus have

or equivalently

$$
\operatorname{Im}\left(\frac{l(t)}{l\left(t_{0}\right)}\right) \begin{cases}>0 & \left(t_{0}<t<t_{0}+\pi\right) \\ <0 & \left(t_{0}+\pi<t<t_{0}+2 \pi\right)\end{cases}
$$

for $t_{0} \leqq t \leqq t_{0}+2 \pi$. Now

$$
\operatorname{Re}\left(\frac{1-e^{2 i\left(t-t_{0}\right)}}{e^{i\left(t-t_{0}\right)}} \frac{l(t)}{l\left(t_{0}\right)}\right) \geqq 0
$$

$$
l(t)=i e^{i t} h^{\prime}\left(e^{i t}\right)-i e^{-i t} \overline{g^{\prime}\left(e^{i t}\right)}
$$

and so (5.11.5) becomes

$$
\operatorname{Re}\left(\left(e^{-i t} e^{i t_{0}}-e^{i t} e^{-i t_{0}}\right) \frac{i e^{i t} h^{\prime}\left(e^{i t}\right)}{l\left(t_{0}\right)}+\left(e^{i t} e^{-i t_{0}}-e^{-i t} e^{i t_{0}}\right) \frac{i e^{i t} g^{\prime}\left(e^{i t}\right)}{\overline{l\left(t_{0}\right)}}\right) \geqq 0 .
$$

Thus on $\{|z|=1\}$,

$$
\operatorname{Re}\left(\left(e^{i t_{0}}-e^{-i t_{0}} z^{2}\right)\left(\frac{i h^{\prime}(z)}{l\left(t_{0}\right)}-\frac{i g^{\prime}(z)}{\overline{l\left(t_{0}\right)}}\right)\right) \geqq 0 .
$$

We now obtain (5.11.1) by applying the maximum principle. 
5.12. Proof of Theorem 5.10. From (5.11.1) we have

$$
e^{-i \mu} h^{\prime}(z)+e^{i \mu} g^{\prime}(z) \ll \frac{1+z}{1-z} \frac{1}{1-z^{2}}=\frac{1}{(1-z)^{2}},
$$

where $\ll$ means that the moduli of the coefficients of the function on the left are bounded by the corresponding coefficients of the function on the right. Hence

$$
e^{-i \mu} h(z)+e^{i \mu} g(z) \ll \frac{z}{1-z} .
$$

We obtain immediately (5.10.1). Next we recall that $g^{\prime}(z)=\omega(z) h^{\prime}(z)$, where $|\omega(z)| \leqq|z|$. From (5.11.1) we obtain

$$
g^{\prime}(z)=\frac{\omega(z)}{e^{-i \mu}+e^{i \mu} \omega(z)} \frac{F(z)}{e^{i \lambda}-e^{-i \lambda} z^{2}},
$$

where $\operatorname{Re} F(z) \geqq 0(|z|<1), F(0)=1$. Thus

$$
g^{\prime}(z) \ll \frac{z}{1-z} \frac{1}{1-z^{2}} \frac{1+z}{1-z}=\frac{z}{(1-z)^{3}} .
$$

This gives (5.10.2). (5.10.3) follows from (5.10.1) and (5.10.2).

5.13. Corollary. If $f \in K_{H}$, then for $n=1,2, \ldots$

$$
\begin{gathered}
\left|a_{-n}(f)\right| \leqq \frac{n-1}{2}+\frac{n+1}{2}\left|a_{-1}(f)\right|, \\
\left|a_{n}(f)\right| \leqq \frac{n-1}{2}\left|a_{-1}(f)\right|+\frac{n+1}{2} .
\end{gathered}
$$

In particular

$$
\left|a_{n}(f)\right|<|n| \quad(|n| \geqq 2) .
$$

Proof. Since the affine transformations (1.2.1) preserve convexity, we can write $f=f_{0}+a_{-1}(f) \bar{f}_{0}$, where $f_{0} \in K_{H}^{0}$. The result of the corollary then follows from Theorem 5.10.

5.14. Convolution theorem. If $f \in K_{H}$ and $\varphi \in K$, then the functions

$$
(\alpha \bar{\varphi}+\varphi) * f \in C_{H} \quad(|\alpha| \leqq 1),
$$

where * denotes convolution.

Proof. Writing $f=\bar{g}+h$ we have

Then for $|\varepsilon| \leqq 1$,

$$
(\alpha \bar{\varphi}+\varphi) * f=\overline{\bar{\alpha}(\varphi * g)}+\varphi * h=\bar{G}+H, \quad \text { say. }
$$

$$
H+\varepsilon G=\varphi *(h+\varepsilon \bar{\alpha} g)
$$


and so, since by (5.7.2), $h+\varepsilon \bar{\alpha} g$ is close-to-convex and $\varphi$ is convex, $H+\varepsilon G$ is close-toconvex by a well-known convolution theorem [8]. Therefore the theorem follows from the following lemma.

5.15. Lemma. Suppose that $G, H$ are analytic in $U$ with $\left|G^{\prime}(0)\right|<\left|H^{\prime}(0)\right|$ and that $H+\varepsilon G$ is close-to-convex for each $\varepsilon(|\varepsilon|=1)$. Then $F=\bar{G}+H$ is harmonic close-to-convex.

Proof. If $F\left(z_{1}\right)=F\left(z_{2}\right)$, then $\bar{G}\left(z_{1}\right)-\bar{G}\left(z_{2}\right)+H\left(z_{1}\right)-H\left(z_{2}\right)=0$. Either $G\left(z_{1}\right)=$ $G\left(z_{2}\right)$ and then $G\left(z_{1}\right)+H\left(z_{1}\right)=G\left(z_{2}\right)+H\left(z_{2}\right)$ so that $z_{1}=z_{2}$ since $G+H$ is univalent; or, with $\varepsilon=\left(\bar{G}\left(z_{1}\right)-\bar{G}\left(z_{2}\right)\right) /\left(G\left(z_{1}\right)-G\left(z_{2}\right)\right)$ we have $\varepsilon G\left(z_{1}\right)+H\left(z_{1}\right)=$ $\varepsilon G\left(z_{2}\right)+H\left(z_{2}\right)$ so that again $z_{1}=z_{2}$. Thus $F$ is univalent since $\left|G^{\prime}(0)\right|<\left|H^{\prime}(0)\right|$.

If $|\alpha|<1,|\varepsilon|=1$, then

$$
\alpha \overline{(H+\varepsilon G)}+H+\varepsilon G
$$

is close-to-convex in $U$ and furthermore maps each circle $\{|z|=r\}(0<r<1)$ onto a close-to-convex curve. Choosing $\alpha=\varrho \varepsilon$ with $0<\varrho<1$ it follows that

$$
L_{\varepsilon}=\varrho \bar{G}+H+\varepsilon(\varrho \bar{H}+G) \quad(|\varepsilon|=1)
$$

is close-to-convex univalent. In particular, writing $z=r e^{i \theta}, \partial L_{\varepsilon} / \partial \theta \neq 0$ and as a function of $z$ is harmonic in $U$. We deduce that

$$
\left|\frac{\partial}{\partial \theta}(\varrho \bar{H}+G)\right|<\left|\frac{\partial}{\partial \theta}(\varrho \bar{G}+H)\right|,
$$

since equality cannot hold, the right-hand expression is non-zero and the inequality is valid at $z=0$. Consider fixed $r(0<r<1)$ and $\theta_{1}, \theta_{2}\left(\theta_{1}<\theta_{2}<\theta_{1}+2 \pi\right)$. If $\Delta$ arg denotes the variation of argument in $\left[\theta_{1}, \theta_{2}\right]$, then, with $|\varepsilon|=1$,

$$
\begin{gathered}
\Delta \arg \frac{\partial}{\partial \theta}(H+\varrho \bar{G}) \\
=\Delta \arg \frac{\partial}{\partial \theta}((H+\varrho \bar{G})+\varepsilon(\varrho \bar{H}+G))-\Delta \arg \left(1+\varepsilon \frac{\frac{\partial}{\partial \theta}(\varrho \bar{H}+G)}{\frac{\partial}{\partial \theta}(H+\varrho \bar{G})}\right) \\
>-\pi-\Delta \arg \left(1+\varepsilon \frac{\varrho \bar{H}_{\theta}+G_{\theta}}{H_{\theta}+\varrho \bar{G}_{\theta}}\right)
\end{gathered}
$$

by the close-to-convexity of $L_{\varepsilon}$ in (5.15.2). Now

$$
\varepsilon \mapsto \frac{1+\varepsilon\left(\varrho \bar{H}_{\theta_{1}}+G_{\theta_{1}}\right) /\left(H_{\theta_{1}}+\varrho \bar{G}_{\theta_{1}}\right)}{1+\varepsilon\left(\varrho \bar{H}_{\theta_{2}}+G_{\theta_{2}}\right) /\left(H_{\theta_{2}}+\varrho \bar{G}_{\theta_{2}}\right)}
$$

maps $\{|\varepsilon|=1\}$ onto a circle with 1 inside and 0 outside, by (5.15.3). Hence $\varepsilon$ with $|\varepsilon|=1$ can be chosen so that the second term on the right of the inequality (5.15.4) is 0 since 
it cannot exceed $\pi$. Thus

$$
\Delta \arg \frac{\partial}{\partial \theta}(H+\varrho \bar{G})>-\pi
$$

and letting $\varrho \rightarrow 1$ we obtain

$$
\Delta \arg \frac{\partial}{\partial \theta} F \geqq-\pi
$$

Since $F$ is univalent, it follows that $F$ maps $\{|z|=r\}$ onto a simple curve bounding a close-to-convex domain. Thus $F(r z)$ is close-to-convex in $U$ for $0<r<1$ and letting $r \rightarrow 1$ the lemma follows by Theorem 3.7.

5.16. Remark. It can be shown that the class of mappings satisfying the conditions of the lemma is invariant under convolutions with mappings of the form $\alpha \bar{\varphi}+\varphi(\varphi \in K)$. An interesting particular case is the following.

5.17. Theorem. Let $f=\bar{g}+h$ be locally univalent in $U$ and suppose that for some $\varepsilon(|\varepsilon| \leqq 1), h+\varepsilon g$ is convex. Then $f$ is univalent close-to-convex.

Proof. If $|\alpha| \leqq 1$, then for suitable real $\mu$,

$$
\operatorname{Re}\left(e^{i \mu} \frac{h^{\prime}(z)+\alpha g^{\prime}(z)}{h^{\prime}(z)+\varepsilon g^{\prime}(z)}\right)>0 \quad(z \in U),
$$

since $\zeta=g^{\prime}(z) / h^{\prime}(z)$ satisfies $|\zeta|<1(z \in U)$ and $\zeta \mapsto(1+\alpha \zeta) /(1+\varepsilon \zeta)$ maps $U$ onto a disc or a half-plane excluding the origin. Consequently $h+\alpha g$ is close-to-convex in $U$ for $|\alpha| \leqq 1$ and so the theorem follows from (5.15).

5.18. If $f$ is harmonic in $U$ we define:

$$
\begin{aligned}
V_{n}(f) & =\left(\begin{array}{c}
2 n \\
n
\end{array}\right)^{-1} \sum_{k=1}^{n}\left(\begin{array}{c}
2 n \\
n+k
\end{array}\right) a_{-k}(f) \bar{z}^{k}+a_{0} \\
& +\left(\begin{array}{c}
2 n \\
n
\end{array}\right)^{-1} \sum_{k=1}^{n}\left(\begin{array}{c}
2 n \\
n+k
\end{array}\right) a_{k}(f) z^{k}
\end{aligned}
$$

as the $n$-th de la Vallée-Poussin mean of $f$. Thus $V_{n}(f)\left(r e^{i t}\right)$ is the $V_{n}$-mean of the Fourier series of $t \mapsto f\left(r e^{i t}\right)$. From Theorem 5.14 we see that $V_{n}(f)$ is close-to-convex, when $f$ is convex, since $V_{n}(z /(1-z))$ is convex [7]. In general it is not true that $\varphi$ convex analytic, $f$ convex harmonic implies $(2 \operatorname{Re} \varphi) * f$ convex harmonic. However, this is true for the $V_{n}$-means.

5.19. Theorem. If $f \in K_{H}$, then $V_{n}(f)$ is convex for $n=1,2, \ldots$ and

$$
V_{n}(f)(U) \subseteq f(U) .
$$

Proof. Since the $V_{n}$ kernels are positive and normalized, (5.19.1) follows from the convexity of $f(U)$. To prove that $V_{n}(f)$ is convex we apply Theorem 3.7, deducing the existence of a sequence $\left(f_{k}\right)$ of convex harmonic functions such that each $f_{k}$ extends smoothly to $\bar{U}$ with $f_{k}(\{|z|=1\})$ a smooth convex curve, and such that 
$f_{k} \rightarrow f(k \rightarrow \infty)$ locally uniformly in $U$. Then it follows from the results of Pólya and Schoenberg [7] that each trigonometric polynomial $V_{n}\left(f_{k}\right)\left(e^{i t}\right)$ maps the unit circle homeomorphically onto a convex curve. Hence applying Choquet's theorem, $V_{n}\left(f_{k}\right)$ is convex in $U$. Since $V_{n}\left(f_{k}\right) \rightarrow V_{n}(f)(k \rightarrow \infty)$ we deduce the convexity of $V_{n}(f)$ from Theorem 5.7.

5.20. Remark. The above argument applies to any variation diminishing kernel $V$ [7]. This property is more crucial here than in the analytic case.

5.21. Remark. The use of Choquet's theorem is also vital for this argument. For example it is not true that $f$ convex in the direction of the real axis implies $V_{n}(f)$ also is, nor even that $V_{n}(f)$ is univalent. For example with $n=2, f=k_{0}$ we have

$$
V_{2}\left(k_{0}\right)(z)=\frac{1}{12} \bar{z}^{2}+\frac{2}{3} z+\frac{5}{12} z^{2}=\overline{g(z)}+h(z),
$$

where $g^{\prime}(z)=z / 6, h^{\prime}(z)=2 / 3+5 z / 6$. Since $h^{\prime}(-4 / 5)=0, V_{2}\left(k_{0}\right)$ is not univalent in $U$. The argument of Theorem 5.19 breaks down only because Choquet's theorem does not extend to cover this case.

5.22. Theorem. Let $f \in C_{H}$. Then

$$
\left|a_{n}(f)\right| \leqq \frac{1}{3}\left(2 n^{2}+1\right) \quad(n= \pm 1, \pm 2, \ldots) .
$$

Equality occurs for the function $k \in \partial C_{H}$.

Proof. Applying the compactness results of Section 3 we may assume that $f$ extends smoothly to $\bar{U}$ with $f(\{|z|=1\})$ a smooth close-to-convex curve $J$. Then on $J$ we have

$$
\Delta \arg d w>-\pi
$$

over any subarc. Writing $f=\bar{g}+h$ this becomes

$$
\begin{gathered}
\arg \left(e^{i \theta_{2}} h^{\prime}\left(e^{i \theta_{2}}\right)-e^{-i \theta_{2}} \overline{g^{\prime}\left(e^{i \theta_{2}}\right)}\right) \\
-\arg \left(e^{i \theta_{1}} h^{\prime}\left(e^{i \theta_{1}}\right)-e^{-i \theta_{1}} \overline{g^{\prime}\left(e^{i \theta_{1}}\right)}\right)>-\pi
\end{gathered}
$$

for $\theta_{1}<\theta_{2}<\theta_{1}+2 \pi$. Also $\left|g^{\prime}\left(e^{i \theta}\right)\right|<\left|h^{\prime}\left(e^{i \theta}\right)\right|$ and so

$$
\begin{gathered}
\Delta \arg \left(e^{i \theta} h^{\prime}\left(e^{i \theta}\right)\right)=\Delta \arg \left(e^{i \theta} h^{\prime}\left(e^{i \theta}\right)-e^{-i \theta} \overline{g^{\prime}\left(e^{i \theta}\right)}\right) \\
-\Delta \arg \left(1-\frac{e^{-i \theta} \overline{g^{\prime}\left(e^{i \theta}\right)}}{e^{i \theta} h^{\prime}\left(e^{i \theta}\right)}\right)>-\pi-\pi=-2 \pi .
\end{gathered}
$$

Thus with $\theta_{1}, \theta_{2}$ fixed we have for arbitrary $\zeta=e^{i \varphi}$,

$$
\left(\theta_{2}+\varphi\right)-\left(\theta_{1}+\varphi\right)+\arg h^{\prime}\left(e^{i\left(\theta_{2}+\varphi\right)}\right)-\arg h^{\prime}\left(e^{i\left(\theta_{1}+\varphi\right)}\right)>-2 \pi
$$

and applying the minimum principle for harmonic functions we deduce

$$
\Delta \arg \left(e^{i \theta} h^{\prime}\left(\zeta e^{i \theta}\right)\right)>-2 \pi \quad(|\zeta|<1) .
$$


In particular when $\zeta=r(0<r<1)$,

$$
\Delta \arg \left(r e^{i \theta} h^{\prime}\left(r e^{i \theta}\right)\right)>-2 \pi \text {. }
$$

This implies that $h^{\prime}$ belongs to the Kaplan class $K(2,4)$, i.e. we can write $z h^{\prime}=S F$, when $S$ is starlike and $|\arg F|<\pi$. We obtain

$$
h^{\prime}(z) \ll \frac{(1+z)^{2}}{(1-z)^{4}},
$$

which is the required result for $n=1,2, \ldots$. If we now assume that $f$ is an extreme point of $\bar{C}_{H}$, then $h^{\prime}$ satisfies $(5.22 .8)$ by approximation and also $f \in \partial \bar{C}_{H}$ applying (2.5) and (2.6). Then $g=e^{i \mu} h$ and the same coefficient estimates hold for $n=-1,-2, \ldots$

5.23. In a forthcoming paper it will be shown that $C_{H}^{0}$ is compact.

\section{Typically real functions}

6.1. The function $f \in H$, the functions harmonic in $U$, is said to be typically real if $f(z)$ is real if, and only if, $z$ is real. To obtain growth conditions we will further assume that $f$ is locally univalent in $U$. If $f=\bar{g}+h$ we suppose that $\left|g^{\prime}(z)\right|<\left|h^{\prime}(z)\right|$ $(z \in U), f(0)=0,\left|h^{\prime}(0)\right|=1$ and $f(r)>0(0<r<1)$. The class of such functions is denoted by $T_{H}$. (Note we do not specify $h^{\prime}(0)=1$.) The subclass of $T_{H}$ with $g^{\prime}(0)=0$ is denoted by $T_{H}^{0}$.

6.2. We observe that, if $f \in S_{H}$ and has real coefficients then $f \in T_{H}$. For $\overline{f(z)}=$ $f(\bar{z})$ and so $\overline{f(z)}=f(z)$ if, and only if, $z=\bar{z}$ because of the univalence.

6.3. We observe further that for $f=\bar{g}+h \in T_{H}$,

$$
\operatorname{Im} f\left(r e^{i \theta}\right) \begin{cases}>0 & (0<\theta<\pi) \\ <0 & (-\pi<\theta<0)\end{cases}
$$

for $0<r<1$. It follows that the analytic function

$$
t(z)=h(z)-g(z)
$$

is typically real. In particular $t(z)$ has real coefficients and so

$$
a_{n}(f)+a_{-n}(f) \in \boldsymbol{R} \quad(n=1,2, \ldots) .
$$

Thus $\overline{a_{1}(f)}-a_{-1}(f) \in \boldsymbol{R}$ and so the function

$$
f_{0}(z)=\frac{\overline{a_{1}(f)} f(z)-a_{-1}(f) \overline{f(z)}}{1-\left|a_{-1}(f)\right|^{2}} \in T_{H}^{0} .
$$


Inverting this we see that

$$
f(z)=a_{1}(f) f_{0}(z)+a_{-1}(f) \overline{f_{0}(z)} .
$$

On the other hand every function of this form with $f_{0} \in T_{H}^{0}$ and

$$
a_{1}(f)+a_{-1}(f)>0, \quad\left|a_{-1}(f)\right|<\left|a_{1}(f)\right|=1
$$

is clearly in $T_{H}$.

6.4. Theorem. If $f_{0} \in T_{H}^{0}$, then $a_{1}\left(f_{0}\right)=1$ and for $n=2,3, \ldots$ we have

$$
\begin{gathered}
|| a_{n}\left(f_{0}\right)|-| a_{-n}\left(f_{0}\right) \mid \leqq n ; \\
\left|a_{-n}\left(f_{0}\right)\right| \leqq \frac{1}{6}(n-1)(2 n-1) ; \quad\left|a_{n}\left(f_{0}\right)\right| \leqq \frac{1}{6}(n+1)(2 n+1) .
\end{gathered}
$$

Equality occurs for the function $k_{0} \in T_{H}^{0}$.

Proof. Since $a_{-1}\left(f_{0}\right)=0$, it is clear that $a_{1}\left(f_{0}\right)$ is real and positive, so $a_{1}\left(f_{0}\right)=1$. Thus the function $t(z)=h_{0}(z)-g_{0}(z)$, where $f_{0}=\bar{g}_{0}+h_{0}$, is a normalized analytic typically real function. Therefore

$$
\left|a_{n}\left(f_{0}\right)-\overline{a_{-n}\left(f_{0}\right)}\right| \leqq n \quad(n=2,3, \ldots)
$$

and we deduce (6.4.1). Also we can write

$$
g_{0}^{\prime}(z)=\omega(z) h_{0}^{\prime}(z),
$$

where $\omega$ is analytic and $|\omega(z)| \leqq|z|(|z|<1)$. We deduce that

$$
g_{0}^{\prime}(z)=\frac{\omega(z)}{1-\omega(z)} t^{\prime}(z) \ll \frac{z}{1-z} \frac{1+z}{(1-z)^{3}}
$$

which gives the first inequality of (6.4.2). The second follows applying (6.4.1).

Applying (6.3.5) we obtain immediately

6.5. Theorem. If $f \in T_{H}$, then

$$
\begin{aligned}
\left|a_{-n}(f)\right| \leqq \frac{1}{6} & \left((n-1)(2 n-1)+\left|a_{-1}(f)\right|(n+1)(2 n+1)\right) \\
& <\frac{1}{3}\left(2 n^{2}+1\right) \quad(n=1,2, \ldots) ; \\
\left|a_{n}(f)\right| \leqq \frac{1}{6} & \left((n+1)(2 n+1)+\left|a_{-1}(f)\right|(n-1)(2 n-1)\right) \\
& <\frac{1}{3}\left(2 n^{2}+1\right) \quad(n=2,3, \ldots) .
\end{aligned}
$$

The function $k \in \partial T_{H}$ is the overall extremal. 


\section{Open questions}

7.1. Problem. If $f=\bar{g}+h$ is univalent in $U$ and real on the real axis with $h^{\prime}(0)+\overline{g^{\prime}(0)}>0$ is it true that $f$ is typically real?

7.2. Harmonic analogue of the Bieberbach conjecture. Let $f \in S_{H}^{0}$. The function $k_{0}$ is on the present evidence the natural candidate for a harmonic Koebe function. Theorem 6.4 suggests the following conjecture:

$$
\begin{gathered}
|| a_{n}(f)|-| a_{-n}(f) \mid \leqq n \quad(n=2,3, \ldots) \\
\left|a_{-n}(f)\right| \leqq \frac{1}{6}(n-1)(2 n-1) \quad(n=2,3, \ldots) .
\end{gathered}
$$

As shown this is true for typically real functions. It is also easily shown using the methods of Section 5 that the conjecture is valid in the cases that $f(U)$ is starlike with respect to the origin or convex in one direction. However, we have no verification in the general close-to-convex case. Furthermore the first inequality is not even proved in the general case for $n=2$.

7.3. Harmonic analogue of the (1/4)-theorem. If $f \in S_{H}^{0}$ we have shown that $\{|w|<1 / 16\} \subseteq f(U)$. What is the best possible constant? For $f=k_{0}$ the constant is $1 / 6$.

7.4. Bounds for inner mapping radius. Find the best lower and upper bounds for the inner mapping radius of $f(U)$ when $f \in S_{H}^{0}$. The lower bound is at least $1 / 16$ by Theorem 4.4.

7.5. Extreme points. Is there a correspondence between the extreme points of $S_{H}^{0}$ and $S$ ?

7.6. Uniqueness. For a given simply connected domain $D$ how unique are the possible mappings $f \in S_{H}, S_{H}^{0}$ with $f(U)=D$ ?

7.7. Multiplier problem. What are the convolution multipliers $\varphi^{*}: K_{H} \rightarrow K_{H}$, i.e. harmonic functions $\varphi$ such that $f \in K_{H} \Rightarrow \varphi * f \in K_{H}$ ? A particular case is the radius of convexity problem: for which values of $r \quad(0<r<1)$ is the function $z \rightarrow f(r z)$ convex in $U$ when $f$ is convex in $U$. Certainly $r \leqq \sqrt{2}-1$.

In conclusion we would like to express our gratitude to three colleagues. Richard Hall for many stimulating conversations and a preview of his recent inequalities for certain Fourier series. Also Richard gave the first example of a univalent harmonic function whose analytic part is not univalent. Aimo Hinkkanen for suggesting the metric giving the constant $1 / 16$. Our original argument gave $1 / 27$. Larry Zalcman for bringing to our attention the work of Choquet on convex maps. 


\section{References}

[1] Choquet, G.: Sur un type de transformation analytique généralisant la représentation conforme et définie au moyen de fonctions harmoniques. - Bull. Sci. Math. (2) 69, 1945, 156165.

[2] Goodman, A. W., and E. B. SafF: On univalent functions convex in one direction. - Proc. Amer. Math. Soc. 73, 1979, 183-187.

[3] Hall, R. R.: On an inequality of E. Heinz. - J. Analyse Math. 42, 1983, 185-198.

[4] Hernz, E.: Über die Lösungen der Minimalflächengleichung. - Nachr. Akad. Wiss. Göttingen Math.-Phys. K1. Math.-Phys.-Chem. Abt., 1952, 51-56.

[5] Lehto, O., and K. I. ViRTAnen: Quasiconformal mappings in the plane. - Springer-Verlag, Berlin-Heidelberg-New York, 1973.

[6] Lewy, H.: On the non-vanishing of the Jacobian in certain one-to-one mappings. - Bull. Amer. Math. Soc. 42, 1936, 689-692.

[7] Pólya, G., and I. J. Schoenberg: Remarks on de la Vallée-Poussin means and convex conformal maps of the circle. - Pacific J. Math. 8, 1958, 295-334.

[8] Ruscheweyh, St., and T. Sheil-Small: Hadamard products of schlicht functions and the PólyaSchoenberg conjecture. - Comment. Math. Helv. 48, 1973, 119-135.

[9] Sheil-Small, T.: On linear accessibility and the conformal mapping of convex domains. - J. Analyse Math. 25, 1972, 259-276.

Open University

Faculty of Mathematics

Milton Keynes

England

Received 26 April 1983
University of York

Department of Mathematics

York

England 